\title{
Análisis de los niveles de estrés en un grupo de maestros con alumnos diagnosticados con trastorno del espectro autista
}

\author{
Analysis of stress levels in a group of teachers with students diagnosed \\ with autism spectrum disorder
}

\begin{abstract}
Resumen
Objetivo y método: se analiza si un grupo de maestros que tienen en el aula alumnos diagnosticados de trastornos del espectro del autismo (TEA) presentan un nivel más elevado de estrés que los que no los tienen. Los participantes $(\mathrm{N}=16)$ proceden de un centro público de la Comunidad Valenciana. El instrumento utilizado es la Escala ED-6. Resultados: el estudio revela que los maestros que enseñan a niños con TEA tienen un nivel de estrés mayor que los maestros que trabajan con niños sin necesidades específicas de apoyo educativo. También se observan diferencias importantes en las variables de ansiedad, creencias y presiones. Conclusiones: tener discentes con TEA puede implicar tener probabilidades de sufrir ansiedad, sentir presiones a lo largo del día escolar, y tener unas creencias que pueden evolucionar negativamente facilitando la aparición de estrés.
\end{abstract}

\section{Palabras clave}

Trastornos del espectro del autismo, TEA, ansiedad, estrés, necesidades educativas especiales, docentes.

\begin{abstract}
Aim and methodology: our aim is to analyse if a group of teachers who have students diagnosed with autism spectrum disorder (ASD) in the classroom have a high level of stress and if, on the other hand, teachers who do not have this type of students do not present stress. Both groups of subjects $(N=16)$ come from a public centre of the Valencian Community. For this purpose, the ED-6 Scale is used. Results: the study reveals that teachers who teach children with ASD have a higher level of stress than teachers who work with children without special education needs. There are also important differences in the variables of anxiety, beliefs and pressures. Conclusions: having students with ASD may imply a chance of anxiety, feeling pressures throughout the school day, and having beliefs that must be controlled due to the possibility of evolving negatively facilitating the emergence of stress.
\end{abstract}

\section{Keywords}

Autism spectrum disorders, ASD, anxiety, stress, special education needs, teachers.

\author{
Marta Díaz Moreno \\ <diaz_marmora@gva.es> \\ CEIP Amanecer de Torrevieja. España
}

\section{Elvira Martínez-Besteiro}

<elvira.martinez@uv.es>

Universitat de València. España

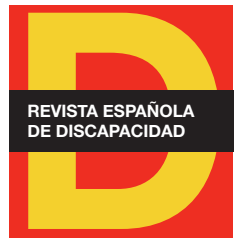

Para citar:

Díaz, M. y Martínez-Besteiro, E. (2019): "Análisis de los niveles de estrés en un grupo de maestros con alumnos diagnosticados con trastorno del espectro autista". Revista Española de Discapacidad, 7 (II): 139-154.

Doi: <https://doi.org/10.5569/23405104.07.02.08>

Fecha de recepción: 04-04-2019 Fecha de aceptación: 24-11-2019 


\section{Introducción}

Desde la psicología cognitiva y con una perspectiva integradora, se considera que el estrés es un "proceso por el cual los eventos ambientales inician una serie de reacciones cognitivas y fisiológicas que finalmente afectan al bienestar" (Ganster y Rosen, 2013: 1088). En esta línea, en los años ochenta, Lazarus y Folkman plantean el modelo transaccional, donde el estrés surge de la relación bidireccional y dinámica que hay entre una persona y su entorno, influyendo en la percepción cognitiva de una exigencia como una amenaza o bien como una oportunidad (Cuevas-Torres y García-Ramos, 2012). Por tanto, el estrés puede ser dañino o no. Cuando se prolonga en el tiempo y tiene una función adaptativa, el estrés es positivo (Ávila, 2014). Sin embargo, en la sociedad actual, el estrés tiene unas connotaciones negativas y se observa una gran incidencia ya que la globalización y la tecnología han aportado comodidades, pero también han afectado al cuerpo y a la mente humana por la severidad y por la rapidez con la que han aparecido en las últimas décadas (Nagra y Arora, 2013). Así, el estrés es considerado como un trastorno psicosocial habitual y suele tener una gran presencia en el ámbito laboral; el $27 \%$ de los trabajadores europeos afirman haber sufrido estrés, ansiedad y/o depresión por el trabajo (European Agency for Safety and Health at Work, 2015). En este sentido, el estrés laboral, u ocupacional, es entendido "como las situaciones en que la persona percibe a su ambiente de trabajo como amenazador para sus necesidades de realización personal o profesional o para su salud física o mental, lo que perjudica su integración en su ámbito de trabajo" (França y Rodrigues, citado en Oswaldo et al. 2012: 70).

Así, dentro del ámbito laboral, la profesión docente suele estar entre las profesiones ocupacionales con mayores casos de estrés (Gonçalves et al., 2015; Hasan, 2014; Health and Safety Executive, 2016). Entre las causas de éste, se citan aspectos como la carga de trabajo, la falta de recursos, las responsabilidades de formar a estudiantes competentes y la necesidad de formarse ellos mismos (Nagra y Arora, 2013). Asimismo, Pagayanan (2016) señala el bajo salario, la falta de materiales para formarse, la presión por conseguir buenos resultados académicos, el mal comportamiento y desinterés de los discentes, y las limitaciones que tienen a la hora de dar reprimendas. Y Karaj y Rapti (2013), resaltan además de algunas de las causas señaladas, el comportamiento de los educandos y las relaciones con la dirección. Por otro lado, Nagra y Arora (2013) evidencian que el profesorado sin alumnos con necesidades educativas, padece estrés 'positivo', pues les permite esforzarse diariamente y Acosta y Burguillos (2014) dan cifras en torno a un $45 \%$ de la presencia de ese estrés positivo en los maestros generales.

Nos centraremos ahora en los maestros que enseñan a escolares con necesidades específicas de apoyo educativo (NEAE) pues son maestros que enseñan a alumnos que precisan una educación adaptada. Dado que el contexto educativo no está totalmente preparado para ofrecer este tipo de educación, ya que vivimos un momento de cambio inclusivo, y que los maestros que trabajan con estos alumnos deben enfrentarse a dificultades relacionadas con sus estudiantes y con las altas expectativas familiares, este tipo de docente puede experimentar soledad, desmotivación, sensación de no reconocimiento, etc. (Cabrera-Mora et al., 2015), aumentando su nivel de estrés. La ambigüedad y el esfuerzo que precisa la nueva realidad educativa, una realidad que lucha por la inclusión como elemento de calidad educativa, puede agravar el estado emocional y psicológico de los maestros. Algunos investigadores han observado que alrededor del $50 \%$ de los maestros que tienen alumnos con NEAE presentan estrés (Ambrosio et al., 2016), debido básicamente al número elevado de estudiantes que había en el aula y a los problemas de comportamiento de los discentes en general; carga de trabajo y problemas de manejo de los discentes que también resaltan Ghani et al. (2014). 
A la hora de comparar la existencia de estrés en maestros con o sin alumnos con NEAE, Mapfumo et al. (2014) declaran que el nivel de estrés de los maestros que tienen alumnos con NEAE es elevado pero que también lo es en los maestros generales, ya que entre ellos no hay diferencias significativas. Contrariamente, Pepe y Addimando (2013) indican que los docentes generales tienen un nivel más alto de estrés que los que trabajan con discentes con NEAE, por la formación que tienen estos últimos. Estos autores también manifiestan que la atención que precisan los niños con NEAE y la agresividad de estos son factores de estrés para su profesorado, aunque influyen menos que la actitud del propio alumno. En este sentido, resaltamos la necesidad de poseer competencias tanto de desarrollo técnico-profesional como de desarrollo socio-personal (Bisquerra y Pérez, 2007), no solo ineludibles para la selección de personal sino también para el desempeño de una buena labor docente. Bajo estas premisas, se puede hipotetizar que el trastorno del espectro autista (TEA) puede influir en el nivel de estrés del docente. Los trastornos del espectro autista son un grupo de trastornos del neurodesarrollo de origen neurobiológico que presentan afectación en la esfera de la comunicación social y de los comportamientos e intereses. Es frecuente, con una prevalencia de uno de cada 68 niños, dura toda la vida y afecta cuatro veces más a los niños que a las niñas (Hervás, 2016: 9). El hecho de que cada caso sea diferente conlleva adaptarse a posibles diferentes realidades individuales de modo que ese esfuerzo adaptativo puede hacer que el profesor se sienta ansioso o estresado (Sultana et al., 2012).

Asimismo, tal como dicen Díaz y Andrade (2015), la inclusión de los alumnos con TEA es difícil puesto que deben luchar contra ciertas barreras relacionadas con el espacio físico y las estrategias didácticas, al mismo tiempo que conviven con sus limitaciones sociales.

En definitiva, tener discentes con TEA en el aula puede ser un gran reto por los sentimientos y reacciones que provoca y por las características que delimitan el trastorno tales como la hipersensibilidad visual y auditiva, la forma particular de percibir el mundo y de procesar la información, etc. (Rangel, 2017). Estos aspectos pueden hacer que cualquier profesor, independientemente de su especialidad y formación, se sienta estresado por la evaluación cognitiva que hace de la situación, afectando tanto a su estado de salud y a su desempeño laboral como al desarrollo educativo de cada niño. En la misma línea, existen investigaciones que alertan de la vertiginosidad con la que la ansiedad, el estrés, la desmotivación y las creencias de autoeficacia negativa surgen desarrollando el síndrome del profesor quemado o de burnout. Si bien, se ha encontrado que tener escolares con NEAE en el aula provoca un afrontamiento optimista ante los problemas (Cabrera et al., 2015). En este sentido, dado que no se sabe ciertamente si el nivel de estrés del profesorado aumenta cuando tiene alumnos que exigen un proceso de enseñanza-aprendizaje adaptado y diferente al resto, se ha estudiado si tener en el aula alumnos con TEA aumenta los niveles de estrés del maestro mediante la administración de la escala de estrés docente ED-6 a una pequeña muestra de docentes procedentes de la Comunidad Valenciana.

\section{Metodología}

Se ha realizado una investigación cuantitativa transversal en una pequeña muestra de maestros para comprobar si el nivel de estrés del profesorado está relacionado con la presencia de alumnos con TEA en el grupo-aula. 


\subsection{Delimitación del problema de estudio}

Puesto que según la literatura el profesorado de Educación Primaria suele padecer síntomas de estrés, independientemente de si tiene o no escolares con NEAE, se estudia si este nivel de estrés es mayor específicamente cuando el profesorado tiene discentes con TEA.

De este modo, el objetivo general del presente estudio es analizar si existe estrés en docentes que tienen alumnos con TEA en el aula. Asimismo, se analiza si dicho estrés es mayor en los maestros con niños diagnosticados de TEA o en maestros sin este grupo de niños en el aula. Además, se analizan las dimensiones de ansiedad, depresión, creencias, presiones, desmotivación y mal afrontamiento y su relación con el aumento del estrés.

\subsection{Participantes}

Participaron 16 maestros de Educación Primaria de un centro público de la Comunidad Valenciana, 13 mujeres y 3 varones, con una edad entre 28 y 52 años $(M=39,43$; $D T=8,5)$. La muestra está dividida en dos grupos: uno formado por ocho maestros con alumnos con TEA y el otro por ocho maestros sin alumnos con TEA.

Los participantes, empleados públicos con al menos cuatro años de experiencia laboral en educación, son representativos de todos los cursos de Educación Primaria, ya que se seleccionó como mínimo un profesor de cada nivel, asimismo todos son tutores e imparten enseñanza directa al alumnado, entre el cual destaca el alumnado con TEA que tiene una permanencia total en el aula ordinaria. La ratio de alumnos con TEA, como establece la normativa educativa, es de uno o dos alumnos con TEA, aunque en la mayoría de los casos la ratio era de un alumno por aula.

\subsection{Instrumento}

Los datos se recogieron por medio de la Escala de estrés docente ED-6 (Gutiérrez-Santander et al., 2005). Cuenta con un total de 77 ítems con una escala Likert de cinco puntos e incluye seis dimensiones: ansiedad, depresión, creencias, presiones, desmotivación y mal afrontamiento. Se ha escogido este instrumento ya que presenta buenas propiedades psicométricas en cuanto a fiabilidad global de la escala (alpha Cronbach=0,90) y consistencia interna en los diversos factores que la componen (Alpha Cronbach=0,74-0,89), como señalan sus autores. Asimismo, en investigaciones más recientes (Samanamud, 2018), se ha corroborado la bondad del instrumento en cuanto consistencia elevada (Alpha Cronbach=0,94), buena estabilidad de la escala $(p<.01)$ de la prueba total y de las subescalas, validez entre la escala y el Inventario de Burnout de Maslach y unos índices de acuerdo del $100 \%$ en claridad y entre el $90 \%$ y el $100 \%$ en relevancia y coherencia con el constructo teórico. Específicamente respecto a las subescalas, presenta una bondad de ajuste elevada ( 0.84 a 0.88 ) en cuanto a ansiedad, depresión y mal afrontamiento. 


\subsection{Procedimiento}

Se administró la Escala ED-6 evitando sesgos en la explicación de cómo cumplimentarla; asimismo, se les informó que la escala era totalmente anónima y se les dio un margen de un mes para entregarla debido a la longitud del instrumento. Posteriormente se recogieron presencialmente los cuestionarios. Se llevaron a cabo análisis descriptivos inferenciales utilizando el SPSS (IBM SPSS Statistics Base 22.0). Todos los maestros seleccionados aceptaron gratamente participar en la investigación y mostraron un amplio interés por descubrir no solo si estaban estresados o no, sino sobre todo por saber qué variables les impedían disfrutar adecuadamente de su docencia.

\section{Resultados}

Se llevó a cabo un análisis de frecuencias y porcentajes y se aplicó la prueba no paramétrica U de MannWhitney debido a las características de la muestra.

Desde el punto de vista descriptivo, se puede observar en la tabla 1 la mayor presencia de estrés en los profesores que tienen alumnos diagnosticados con TEA. Para una mayor comprensión de los resultados, los niveles se han divido en dos categorías: presencia (nivel alto y moderado) o ausencia (nivel normal y bajo) de estrés negativo.

Tabla 1. Existencia o no de estrés en ambos grupos según la Escala ED-6

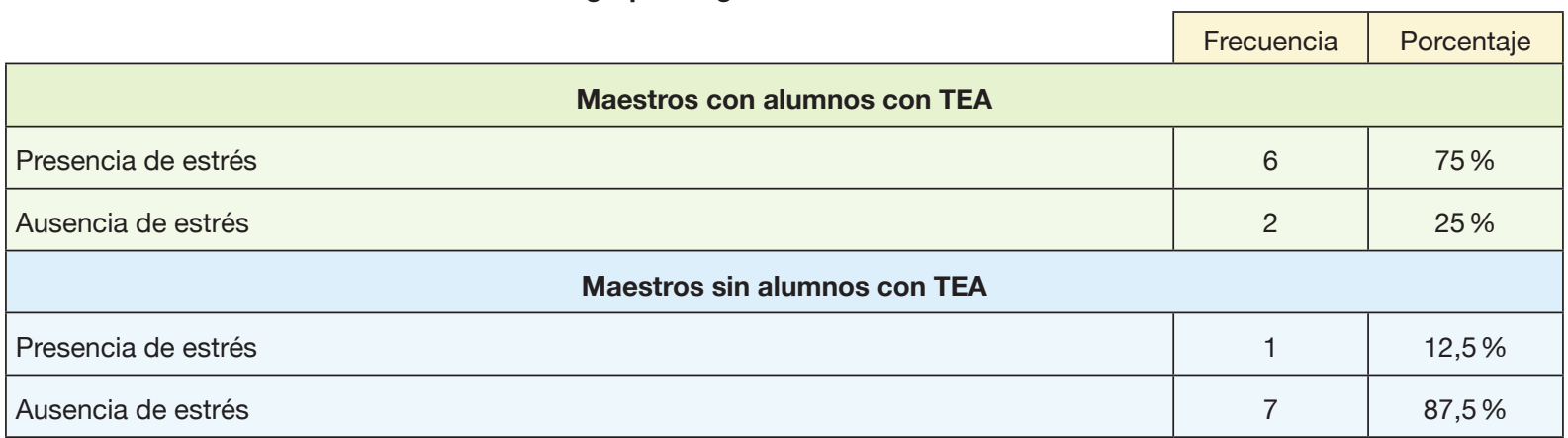

Fuente: elaboración propia.

Así, en la tabla 2 se observa que el grupo de maestros que tiene estudiantes diagnosticados con TEA suele tener un nivel moderado de estrés. Al contrario, la mayoría de los docentes que no tienen escolares diagnosticados con TEA en su aula tienen un nivel normal de estrés. 
Tabla 2. Niveles de estrés de los maestros de ambos grupos

\begin{tabular}{|c|c|c|c|c|}
\hline \multicolumn{2}{|c|}{ Maestros con alumnos con TEA } & \multicolumn{2}{c|}{ Maestros sin alumnos con TEA } \\
\hline Niveles & Frecuencia & Porcentaje & 1 & Frecuencia \\
\hline Bajo & 1 & $12,5 \%$ & 6 & $12,5 \%$ \\
\hline Normal & 1 & $12,5 \%$ & 1 & $75 \%$ \\
\hline Moderado & 5 & $62,5 \%$ & 0 & $12,5 \%$ \\
\hline Alto & 1 & $12,5 \%$ & 0 \\
\hline
\end{tabular}

Fuente: elaboración propia.

Como puede observarse en la tabla 3 existen algunas diferencias significativas con un tamaño del efecto de medio a grande. En referencia al estrés global, los grupos difieren significativamente tanto por niveles $(p<.05)$ como por puntuaciones directas $(p<.05)$. Respecto a las dimensiones del cuestionario se han encontrado diferencias significativas respecto a ansiedad $(p<.05)$, creencias $(p<.05)$ y presiones $(p=.01)$.

Tabla 3. Prueba U de Mann-Whitney

\begin{tabular}{|c|c|c|c|c|}
\hline \multirow{2}{*}{\multicolumn{2}{|c|}{ Dimensiones }} & $\begin{array}{c}\text { Maestros con alumnos } \\
\text { con TEA }\end{array}$ & $\begin{array}{c}\text { Maestros sin alumnos } \\
\text { con TEA }\end{array}$ & \multirow{2}{*}{$\begin{array}{c}\text { Sign. Asintót. } \\
\text { Bilateral } \\
\text { (Tamaño del efecto) }\end{array}$} \\
\hline & & $\begin{array}{l}\text { Suma de rangos } \\
\text { (rango promedio) }\end{array}$ & $\begin{array}{l}\text { Suma de rangos } \\
\text { (rango promedio) }\end{array}$ & \\
\hline \multicolumn{2}{|c|}{ Ansiedad } & $86(10,75)$ & $50(6,25)$ &, $04(r=0,49)$ \\
\hline \multicolumn{2}{|c|}{ Depresión } & $69(8,63)$ & $67(8,38)$ & ,90 \\
\hline \multicolumn{2}{|c|}{ Creencias } & $84(10,50)$ & $52(6,50)$ &, $02(r=0,56)$ \\
\hline \multicolumn{2}{|c|}{ Presiones } & $90(11,25)$ & $46(5,75)$ &, $01(r=0,62)$ \\
\hline \multicolumn{2}{|c|}{ Desmotivación } & $59(7,38)$ & $77(9,63)$ & 31 \\
\hline \multicolumn{2}{|c|}{ Mal afrontamiento } & $65(8,13)$ & $71(8,88)$ & ,72 \\
\hline \multirow{2}{*}{ Estrés } & Por niveles & $86(10,75)$ & $50(6,25)$ &, $04(r=0,51)$ \\
\hline & Por PD & $89,50(11,19)$ & $46,50(5,81)$ &, $02(r=0,57)$ \\
\hline
\end{tabular}

Nota. PD= Puntuación directa.

Fuente: elaboración propia.

En la tabla 4 se observan los niveles de estrés de los participantes en forma de porcentaje teniendo en cuenta las dimensiones que forman el estrés según el instrumento. 


\begin{tabular}{|c|c|c|}
\hline \multirow{2}{*}{ Dimensiones del estrés } & Maestros con alumnos con TEA & Maestros sin alumnos con TEA \\
\hline & Nivel de estrés (porcentaje) & Nivel de estrés (porcentaje) \\
\hline \multirow{2}{*}{ Ansiedad } & Alto $(37,5 \%)$ & \multirow{2}{*}{ Normal (50\%) } \\
\hline & Moderado $(37,5 \%)$ & \\
\hline Depresión & Bajo $(62,5 \%)$ & Bajo (62,5\%) \\
\hline \multirow{2}{*}{ Creencias } & Normal (50\%) & \multirow{2}{*}{ Bajo (100\%) } \\
\hline & Bajo (50\%) & \\
\hline Presiones & Moderado $(62,5 \%)$ & Bajo (50\%) \\
\hline Desmotivación & Normal (50\%) & Normal $(37,5 \%)$ \\
\hline Mal afrontamiento & Moderado (62,5\%) & Moderado (50\%) \\
\hline
\end{tabular}

Fuente: elaboración propia.

A continuación, señalaremos los ítems más relevantes, según las diferencias significativas encontradas en cada dimensión analizada y según el nivel de estrés respecto a dichas dimensiones.

En la variable de ansiedad, la mayoría de los maestros con alumnos con TEA padecen ansiedad a un nivel alto y moderado debido a la tensión que sienten durante la jornada laboral (ítem 6) y a los problemas de sueño (ítem 6). Ninguno de ellos tiene síntomas físicos (ítem 11). Los maestros que no tienen niños con NEAE consideran que deberían de actuar con más calma en el trabajo (ítem 14) y que se dan situaciones de tensión con las que se les acelera el pulso (ítem 19). En ambos grupos es habitual el consumo de sustancias para aliviar el malestar (ítem 2).

En cuanto a las creencias, parece ser especialmente importante la presencia de creencias irracionales en relación con las exigencias de la política (ítem 36) y la falta de valoración social de la labor docente (ítem 32). También los maestros con alumnos con TEA revelan tener creencias desadaptativas sobre el salario (ítem 31) y las responsabilidades parentales (ítem 39).

Asimismo, un porcentaje considerable de docentes con alumnos con TEA sitúan las presiones en un nivel moderado por la dificultad que suponen las adaptaciones curriculares (ítem 44), debido a las frecuentes riñas (ítem 46) y por el comportamiento de algunos alumnos durante las sesiones (ítem 49). Contrariamente, los maestros sin discentes con NEAE revelan que en sus aulas hay un buen clima de aprendizaje (ítem 44) y que no riñen a los alumnos porque no suelen molestar (ítems 46 y 49).

Respecto al resto de dimensiones, aunque no se han observado diferencias significativas, destacamos algunas cuestiones en cuanto al análisis de los ítems desde el punto de vista cualitativo.

La mitad de la muestra puntúa la desmotivación en un nivel normal, aunque confiesan que los malos momentos personales de los discentes afectan (ítem 64). En el caso de los maestros sin alumnos con TEA, estos consideran que no conservan la ilusión que tenían en sus inicios laborales (ítem 58). 
El mal afrontamiento se corresponde con un nivel moderado de estrés, que se explica por los problemas para animarse cuando se está triste (ítem 66), la falta de recursos (ítem 74), la no facilidad para resolver los problemas del trabajo (ítem 71) y la percepción de que el aula y la organización del centro no es adecuada (ítem 67 y 70). Los maestros sin alumnos con TEA puntúan en mal afrontamiento a un nivel moderado, debido a la falta de recursos (ítem 74) y a las difíciles relaciones con los superiores (ítem 69). Ninguno de los dos grupos busca ayuda ni apoyo cuando tienen problemas laborales (ítem 76), y no dan demasiada importancia a las relaciones sociales (ítem 72 y 73 ).

\section{Discusión}

El objetivo principal de este estudio es analizar la existencia de estrés en una muestra de maestros con y sin alumnos con TEA en el aula.

Resaltamos que la existencia de estrés 'negativo', conlleva síntomas que pueden afectar a la salud y al rendimiento personal (Echeburúa, 2016), bloqueando al individuo en varias facetas de la vida pudiendo llegar a desarrollar burnout (Ruiz-Calzado, 2016; Ruiz-Calzado y Llorent, 2018; Vieira y Sacani, 2014). En este sentido, tal como se ha podido observar, se verifica la hipótesis de este estudio, es decir, se concluye que el nivel de estrés está relacionado con la presencia de discentes con TEA, ya que los docentes de este grupo tienen estrés y a un nivel más elevado que aquellos que no tienen escolares que requieren tanta atención. Estos resultados van en la línea de Ambrosio et al. (2014); Ferreira y Alves (2016); Ghani et al. (2014) y Lazuras (2006) quienes observaron que el profesorado que enseña a estudiantes con NEAE tienen un nivel moderado de estrés, y de Acosta y Burguillos (2014) y Nagra y Arora (2013), en cuanto a que los maestros que no educan a alumnos con TEA, o con cualquier otra NEAE, tienen un nivel normal de estrés. Así, el nivel normal de estrés ('adaptativo') mejora la salud física, hace que el sujeto tenga pasión y motivación, y reduce otros tipos de estrés (Venkatesh y Ram, 2015).

Sin embargo, hay otros estudios que indican lo contrario, tales como Gonçalves et al. (2014), Hasan (2014) y Mapfumo et al. (2014), Pagayanan (2016). Pensamos que es una diferencia de tipo sociocultural, político y por las condiciones educativas de dichos estudios. En el caso de Mapfumo et al. (2014), el nivel de estrés era elevado en todos los tipos de maestros en Zimbabue, con o sin alumnos con NEAE; el resto de los estudios señalados se han llevado a cabo en países como Brasil, India y Filipinas. Asimismo, el tipo de muestra también puede influir en los resultados del estudio de Pepe y Addimando (2013). Estos declaran que los maestros de niños sin NEAE (niños con problemas de conducta) tienen un estrés mayor que los maestros de escolares con NEAE (niños que tienen diferentes necesidades educativas de grado leve y problemas de conducta) por la formación que tiene estos últimos. Se parte de la idea de que los profesores ordinarios de su muestra son poco tolerantes con las situaciones estresantes, lo que provoca que el nivel de estrés de estos aumente con facilidad.

Comentamos a continuación los resultados respecto al análisis de las dimensiones. 
En primer lugar, en cuanto a la variable de ansiedad, se han encontrado diferencias significativas entre los dos grupos, con un tamaño del efecto medio $(r=.0,49)$ lo cual demuestra que los maestros con discentes con TEA pueden tener mayores probabilidades de padecerla y a un nivel moderado y alto. Al respecto, Muñoz y Lucero (2013) advierten de que los docentes que trabajan con niños con NEAE pueden padecer ansiedad, pero no especifica a qué nivel de gravedad. Según los ítems más valorados, los niveles obtenidos se deben a la tensión que experimenta este tipo de maestro durante la jornada laboral, tensión que según Prat (citado en Acosta y Burguillos, 2014) es común en todo el colectivo docente. Esta tensión podría provenir, tal como dicen Vesga y Vesga (2015), de la falta de conocimiento de estrategias pedagógicas para tratar adecuadamente a estos discentes, porque el sistema educativo actual intenta introducir inclusivamente a estos niños en las aulas ordinarias, pero no suele ofrecer cursos formativos concretos para el profesor que va a tener en su aula a un alumno con NEAE, sino que más bien organiza cursos de inclusión general. La tensión que le invade le afecta sobre todo a nivel psíquico, de ahí que gran parte de la muestra experimente problemas de sueño. En este sentido, Amezcua-Sandoval et al. (2011a) descubrieron que el 49,6\% de su muestra (maestros que trabajaban con estudiantes con NEAE) tenían al menos un síntoma de trastornos del sueño. Paralelamente, se desvela que el profesorado que no tiene alumnos con TEA presenta un nivel normal de ansiedad, un nivel que no interfiere en su cometido laboral, lo cual es concordante con la investigación de Linares y Gutiérrez (2014) y Merchán y González (2014).

En segundo lugar, respecto a la variable de depresión, en nuestro estudio no hemos observado diferencias significativas, resultados que ya mencionan Amezcua-Sandoval et al. (2011b), pues confirman la existencia de un porcentaje bajo de depresión en los maestros que tienen niños de educación especial. No obstante, algunas investigaciones sí resaltan que los profesores presentan depresión. En el trabajo de Muñoz y Lucero (2013), se menciona que la mitad de su muestra (maestras de educación especial) tienen riesgo de padecer depresión, resultados que pueden deberse a que son maestras de discapacidades múltiples, maestras que trabajan con muchos tipos de NEAE, entre ellas el autismo de grado severo, y no de grado leve (este grado es el que hay en el contexto del estudio). Y Vidal et al. (2013), concluyen que un poco más de la mitad de su muestra (profesores generales de Primaria) tiene depresión, conclusiones que pueden deberse al hecho de que estos investigadores cuentan con una muestra muy amplia, de 414 personas, y que extraen la información de la Classificação Estatística Internacional de Doenças e Problemas Relacionados com a Saúde - CID 10 (sería el equivalente a la CIE-10 española), mientras que el presente estudio tiene una muestra menos representativa y la depresión se evalúa en diez ítems que hacen referencia a los síntomas más habituales en el sector educativo.

En tercer lugar, señalamos las creencias desadaptativas. Se han observado diferencias significativas respecto a las creencias de los dos grupos, con un tamaño grande del efecto $(r=0,56)$. Estas diferencias aluden a que hay profesores que tienen creencias irracionales pero que estas no son un obstáculo para que reflexionen sobre la propia actitud o la enseñanza, especialmente cuando hay aspectos que perjudican al logro de los propósitos educativos, tal como indica González (2012). Habría que tener en cuenta, como dice Rangel (2017), el reto que implica tener estudiantes con TEA, especialmente si no se tiene una buena experiencia y formación al respecto; indicamos como ejemplo la necesidad de formación para evaluar la teoría de la mente tanto en docentes como en familias (Galende et al., 2012). Ninguno de nuestros participantes, a partir de los resultados, demuestra tener creencias irracionales que afecten a su desempeño laboral y a su nivel de estrés, resultado que no concuerda con la investigación de Popov et al. (2015), quienes consideran que las creencias influyen considerablemente en el estrés de los maestros según sus niveles de autodestrucción, 
autoritarismo, demandas de justicia y baja tolerancia a la frustración. Nuevamente, destacamos la posible intervención de aspectos socioculturales y de procedimiento metodológico, pues este estudio se llevó a cabo en Serbia utilizando la Teacher Irrational Beliefs Scale (TIBS), la Sources of Work Stress Scale (IRS) y la Depression, Anxiety and Stress Scale (DAS-S 21). En nuestra investigación, los dos grupos estudiados consideran que el trabajo docente está muy mal valorado en la sociedad, conclusiones a las que llegan Cabrera et al. (2015) y la Organisation for Economic Co-operation and Development (OECD) (2014). También piensan que la política educativa exige mucho más de lo que ofrece, aspecto que concuerda con los datos de Cabrera et al. (2015). Estas declaraciones reflejan a un colectivo que no se siente recompensado socialmente como cree que se merece, que no recibe el apoyo social y administrativo que necesita para poder enfrentarse al día a día. Igualmente, es curioso que aparte de estas declaraciones, los maestros que tienen escolares con TEA manifiesten un descontento hacia el salario recibido y hacia la actitud de los padres, los cuales son exigentes y no asumen su responsabilidad parental en cuestiones educativas. Ramírez et al. (2012) mencionan que tanto el salario como la constante supervisión que hacen los padres sobre la labor del docente son grandes estresores para los maestros, lo cual se puede intuir en aquellos que son inclusivos, ya que estos deben lidiar con las circunstancias educativas y con el control de padres que no acaban de comprender la situación de su hijo o que se niegan a aceptarla.

En cuarto lugar, en referencia a la variable de presiones, el estudio muestra que hay diferencias significativas, con un tamaño grande del efecto $(r=0,62)$ entre los dos grupos que componen la muestra. Los maestros con alumnos con TEA se sienten presionados constantemente y a un nivel preocupante, mientras que los docentes que no tienen niños con estas NEAE no perciben presiones durante su jornada laboral. Deberíamos considerar que cada uno de los dos grupos explica la vida escolar desde diferentes perspectivas y exponen unas circunstancias escolares muy distintas, como si de otra realidad escolar se tratara. Los docentes que enseñan a niños con TEA exteriorizan que se sienten muy presionados durante la jornada laboral, deseando incluso que esta acabe antes de lo previsto, porque se sienten extenuados y agotados debido al tiempo que utilizan en dar reprimendas a los alumnos, resultados que coinciden con lo expuesto por Ghani et al. (2014) y por Marchesi y Díaz (2007). En contraposición, los docentes que no tienen discentes diagnosticados con TEA confiesan que hay un buen clima de aprendizaje en el aula y que no utilizan mucho tiempo en reñir a los alumnos, de manera que pueden desarrollar sus labores sin ninguna presión y, por consiguiente, no sienten la necesidad de que el día o el curso académico acabe antes de lo esperado. También es interesante destacar que los maestros que tienen escolares con TEA puntúan altamente como presión realizar adaptaciones curriculares, las cuales son presiones que no suelen experimentar los profesores que no tienen un alumno con NEAE. En esa línea, Horne y Timmons (2009) confirman que los docentes reclaman que en los centros haya profesores de apoyo que ayuden a realizar las adaptaciones curriculares. En cualquier caso, se observa que los maestros inclusivos necesitan ayuda, ya sea por falta de conocimientos en cuanto a la creación de estas o por falta de tiempo y recursos para llevarlas a cabo. Darle este tipo de ayuda generaría una actitud más positiva y de mayor disposición hacia la atención a la diversidad (Granada et al., 2013). Aunque no existen investigaciones concretas sobre las presiones y la existencia o no de las mismas en los maestros con o sin estudiantes con NEAE, algunos autores que han estudiado el estrés en estos dos tipos de docentes exponen que tienen presiones relacionadas con la carga de trabajo, el tiempo (Karaj y Rapti, 2013), los resultados académicos (Pagayanan, 2016) y la falta de material y recursos (Ghani et al., 2014). Como se ha podido observar, los participantes del estudio no manifiestan ninguna de estas presiones como tal, pero sí podrían están inmersas en aspectos de otras variables del estrés como la falta de recursos en el mal afrontamiento. 
En quinto lugar, no se han hallado diferencias significativas entre los grupos en la variable de desmotivación, ya que las puntuaciones están equilibradas y ninguna persona de la muestra se siente desmotivada. No obstante, analizando exhaustivamente los niveles de estrés se observa que, a diferencia de las anteriores variables, los docentes que tienen discentes con TEA se encuentran en una mejor situación que el otro grupo, porque todos los sujetos, a excepción de una persona, están motivados. En parte, estos resultados pueden ser explicados por el trabajo mental desafiante que tienen que hacer constantemente los maestros que tienen alumnos con TEA para afrontar las situaciones y no decaer ante las presiones. Es este trabajo mental el factor que más influye en la satisfacción laboral (Chamundeswari, 2013), la cual está estrechamente relacionada con la motivación (Jiménez et al. 2016). Además, Silva y Valderrama (2016) muestran que los profesores que tienen niños de educación especial tienen motivación para ayudarlos y para que aprendan, puesto que confirman que tienen una actitud muy favorable y de colaboración que los lleva a querer actuar incluso fuera del aula. Ahora bien, el grado de motivación de este tipo de maestros es muy sensible, pues cualquier factor puede convertirlo en desmotivación (Cabrera et al. 2015). Por el contrario, aquellos que no tienen alumnos con TEA son una muestra más heterogénea, aunque el porcentaje de personas que no están desmotivadas es elevado. Estos últimos resultados son congruentes con los indicados por Ávalos y Sotomayor (2012) y por la OECD (2014). Sin embargo, aunque se desconoce cuál es el grado de motivación de todos los participantes, todos tienen un estado de ánimo normal, realista, ya que son conscientes de los aspectos negativos, afectándoles cuando realmente deben hacerlo, al mismo tiempo que se sienten capacitados para luchar con cierto entusiasmo y no desvanecerse. Murillo y Román (2012) concluyen que el sentido de pertenencia al contexto escolar interviene en el nivel de motivación. Por tanto, estos dos grupos pueden tener una motivación normal por ese sentimiento de pertenencia, sentimiento que es neutro. Ningún docente da indicios de que la vida escolar que llevan no sea de su agrado o que se sientan incómodos en la misma. La motivación es un elemento indispensable para poder afrontar las situaciones y, consecuentemente, incide en la disminución del estrés. Como señalan Accariya y Khalil (2016), a medida que el nivel de estrés aumenta, el nivel de desmotivación también lo hace. Cabe destacar que Canrinus et al. (2012) consideran que el grado de motivación varía según las creencias y las emociones que se tienen. En este sentido, los participantes no tienen creencias desadaptativas que influyan en su vida laboral, lo cual puede estar determinando el nivel de motivación que presentan, ya que al no tener ideas preconcebidas sobre la educación o el proceso educativo, no se sienten con limitaciones emocionales a la hora de actuar educativamente.

En sexto y último lugar no hemos encontrado diferencias significativas entre los dos grupos respecto a la variable mal afrontamiento, aunque en ambos casos los resultados son negativos. Los dos grupos que forman la muestra tienen problemas para enfrentarse a las situaciones educativas, aunque se observa, por la frecuencia en los niveles de estrés, que los maestros que tienen estudiantes con TEA tienen un peor afrontamiento. Según Runes (2010: 218) "el afrontamiento sólo es posible a través del fortalecimiento de los vínculos sociales entre docentes", lo cual explica que toda la muestra tenga problemas de afrontamiento, puesto que los participantes no dan demasiada importancia a las relaciones sociales, especialmente con sus compañeros. Ambos grupos consideran que no disponen de los recursos necesarios para desempeñar sus labores como creen, lo cual es un estresor en la muestra de maestros generales que estudia Nagra y Arora (2013). Así pues, como indican Ramírez et al. (2012), el profesorado percibe este factor como un gran estresor, ya que al no poder controlar el mismo, se sienten angustiados y no saben cómo solucionar la situación. También la muestra del presente estudio menciona que no buscan ayuda social o consejos cuando tienen problemas laborales, lo cual según Sandín (citado en Echeburúa, 2016) perjudica al afrontamiento del estrés. 
Todos estos datos muestran que tener discentes con TEA puede implicar una mayor probabilidad de sufrir ansiedad, sentir presiones a lo largo del día escolar y tener unas creencias que no influyen dramáticamente en el estrés, pero sí que se deben tener controladas debido a la posibilidad de evolucionar negativamente facilitando la aparición de estrés.

\section{Conclusiones y limitaciones}

Este estudio pretende tener una finalidad preventiva e informativa sobre la necesidad de indagar determinadas variables y su relación con el estrés entre el colectivo docente, puesto que la educación y calidad de vida de muchos niños está bajo las actuaciones de estos maestros. Del mismo modo, el estudio refleja la necesidad de que el sistema educativo tenga docentes formados y capacitados para atender a todos los escolares, porque es imposible conseguir una educación inclusiva si los encargados de hacerla funcionar no tienen la competencia necesaria para ello. Igualmente, deben saber cómo afrontar la sobrecarga de trabajo y las circunstancias que se van dando en el contexto escolar. Como dice Echeburúa (2016), el reto de la educación debe ser dar a todas las personas que están inmersas en ella las habilidades personales necesarias para aumentar la resistencia al estrés y el umbral al mismo, eliminando así el estrés innecesario.

Sin embargo, debido al tamaño de la muestra y que no se ha realizado un muestreo probabilístico, no se pueden generalizar los resultados, aunque las submuestras difieren significativamente y los resultados parecen ir en la línea de diversos autores si tenemos en cuenta algunas posturas encontradas influidas probablemente por factores socioculturales y metodológicos. Se aconsejan estudios en profundidad de las variables analizadas tales como ansiedad o depresión con instrumentos más específicos y estudiar si se relacionan con el estrés de los docentes. Por otro lado, si se replicara este estudio con una muestra mayor, habría que analizar también las posibles diferencias entre sexos, ya que en algunas investigaciones se ha observado que el nivel de estrés percibido de las mujeres es mayor que el de los hombres (Avci et al., 2017). Del mismo modo, el concepto que tiene cada participante de lo que es un problema es una limitación, y en este sentido, una futura investigación podría ir en la línea de clarificar y operativizar de algún modo dicho concepto. 


\section{Referencias bibliográficas}

Accariya, Z. y Khalil, M. (2016): "The relations between management style, work motivation and feeling of stress among the arab School Community”. Creative education, (7): 1995-2010.

Acosta, M. y Burguillos, A. I. (2014): "Estrés y burnout en profesores de Primaria y Secundaria de Huelva: Las estrategias de afrontamiento como factor de protección". INFAD, Revista de Psicología, 4 (1): 303-310.

Ambrosio, K. et al. (2014): "Indicadores de estresse e coping no contexto da educação inclusiva". Educação e Pesquisa, 40 (1): 127-142.

Amezcua-Sandoval, M. T. et al. (2011a): "Factores psicosociales laborales y trastornos de sueño en docentes de educación especial”. Revista Educación Inclusiva, 4 (2): 19- 26.

Amezcua-Sandoval, M. T. et al. (2011b): "Factores psicosociales y sintomatología depresiva en profesionales docentes que trabajan con alumnos especiales". Revista de Educación y Desarrollo, (19): 67-72.

Ávalos, B. y Sotomayor, C. (2012): "Cómo ven su identidad los docentes chilenos". Perspectiva Educacional, 51 (1): 77-95.

Avci, A. et al. (2017): "Psychological Needs as the Predictor of Teachers' Perceived Stress Levels". Journal of Education and Training Studies, 5 (4): 154-164.

Ávila, J. (2014): “El estrés un problema de salud del mundo actual”. Revista conciencia, 2 (1): 117-125.

Bisquerra Alzina, R. y Pérez Escoda, N. (2007): “Las competencias emocionales”. Educación XX1, 10: 61-82.

Cabrera-Mora, L. et al. (2015): "La percepción del docente acerca de sí mismo en la práctica de la educación especial”. Revista de Educación y Desarrollo, (33): 39-43.

Canrinus, E. T. et al. (2012): "Self-efficacy, job satisfaction, motivation and commitment: exploring the relationships between indicators of teachers' professional indentity”. Eur JPsychol Educ (27): 115-132.

Chamundeswari, S. (2013): "Job satisfaction and performance of school teachers". International Journal of Academic Research in Business and Social Sciences, 3 (5): 420-428.

Cuevas-Torres, M. y García-Ramos, T. (2012): “Análisis crítico de tres perspectivas psicológicas de estrés en el trabajo". Trabajo y Sociedad, (19): 87-102.

Díaz, E. y Andrade, I. (2015): "El Trastorno del Espectro Autista (TEA) en la educación regular: estudio realizado en instituciones educativas de Quito, Ecuador”. Revista Intercontinental de Psicología y Educación, 17(1): 163181.

Echeburúa, E. (2016): "Estrés y recursos de afrontamiento en la sociedad contemporánea". Avances en Salud Mental Relacional, 15 (1): 1-10.

European Agency for Safety and Health at Work (2015): Manage stress (en línea). <https://osha.europa.eu/en/ tools-and-publications/publications/reports/healthy-workplaces-good-practice-awards-2014-2015>, acceso 22 de mayo de 2017.

Ferreira, M. R. T. y Alves, Z. V. (2016): "O poder preditivo do coping e do engagement no stresse laboral dos professores do ensino especial”. Informes Psicológicos, 16 (2): 13-25. 
Galende Pérez, N. et al. (2012): "Nuevas perspectivas en torno a la teoría de la mente: aplicación práctica en el ámbito familiar y escolar". Educación XX1, 15 (2).

Ganster, D. C. y Rosen, C. C. (2013): "Work Stress and Employee Health: A Multidisciplinary Review". Journal of Management, 39 (5): 1085-1122.

Ghani, M. Z. et al. (2014): "Stress among special education teachers in Malaysia". Procedia-Social and Behavioral Sciences, 114: 4-13.

Gonçalves, G. B. et al. (2015): "Assessment of Stress, Depressive, and Anxiety Symptoms in Teachers in the Public Education Network". Annals of Depression and Anxiety, 2 (3): 1-6.

González, C. (2012): "Las creencias del profesor que imparte asignaturas en la escuela normal”. Atenas, Revista Científico Pedagógica, 3 (18): 117-125.

Granada, M. et al. (2013): “Actitud de los profesores hacia la inclusión educativa”. Papeles de Trabajo, 25: 51-59.

Gutiérrez-Santander, P. et al. (2005): "El estrés docente: elaboración de la escala ED- 6 para su evaluación”. RELIEVE, 11 (1): 47-61.

Hasan, A. (2014): "A study of occupational stress of Primary school teachers”. Educationia Confab, 3 (4): 11-19.

Health and Safety Executive. (2016): Work related Stress, Anxiety and Depression Statistics in Great Britain 2016 (en línea). <http://www.hse.gov.uk/statistics/causdis/stress.pdf>, acceso 15 de mayo de 2017.

Hervás, A. (2016): “Un autismo, varios autismos. Variabilidad fenotípica en los trastornos del espectro autista”. Neurología, (62): 9-14.

Horne, P. E. y Timmons, V. (2009): "Making it work: teachers' perspectives on inclusion”. International Journal of Inclusive Education, 13 (3): 273-286.

Jiménez, A. et al. (2016): “¿Satisfacción por vocación?: La influencia del tipo de trabajo en la satisfacción laboral”. ReiDoCrea, (5): 90-96.

Karaj, S. y Rapti, E. (2013): “Teacher job stress in Albania: Examining the role of students' classroom disruptive behavior and other factors in the school context". Problems of education in the $21^{\text {st }}$ century, (54): $14-21$.

Lazuras, L. (2006): "Occupational stress, negative affectivity and physical health in special and general education teachers in Greece". British Journal of Special Education, 33 (4): 204-209.

Linares, O. L. y Gutiérrez, R. E. (Julio de 2014): Beneficios del apoyo social y del trabajo ante la reacción de estrés y ansiedad en profesores. Las ciencias humanas en el marco de la sociedad de la información y del conocimiento. Conferencia llevada a cabo en el II Congreso Internacional de Psicología y Humanidades de la Universidad de Guadalajara, Jalisco (México).

Mapfumo, J. et al. (2014): "Sources and levels of stress among mainstream and special needs education teacher in Mutare Urban in Zimbabwe". Int J EduSci, 6 (2): 187-195.

Marchesi, A. y Díaz, T. (2007): "Las emociones y los valores del profesorado". Cuadernos Fundación SM, (5): 1-127.

Merchán, I. M. y González, J. D. D. (2014): “Autopercepción de la ansiedad en maestros de Primaria de Badajoz y Castelo Brando". INFAD, Revista de Psicología, 2 (1):177-186.

Muñoz, M. T. y Lucero, B. A. (2013): "Calidad de vida profesional de trabajadoras de una escuela de estudiantes con discapacidades múltiples”. Salud de los Trabajadores, 21 (2): 151-162. 
Murillo, F. J. y Román, M. (2012): "Formación, motivación y condiciones laborales de los docentes de Primaria en Perú”. Revista Peruana de Investigación Educativa, (4): 7- 42.

Nagra, V. y Arora, S. (2013): "Occupational stress and health among teacher educators". International Journal on Advanced Research in Management and Social Sciences, 2 (8): 1-13.

Organisation for Economic Co-operation and Development (OECD) (2014): Teaching infocus (en línea). <https:// www.oecd.org/edu/school/TIF6.pdf>, acceso 22 de mayo de 2017.

Oswaldo, Y. C. et al. (2012): "Impacto de los estresores laborales en los profesionales y en las organizaciones. Análisis de investigaciones publicadas". Invenio, 15 (29): 67- 80.

Pagayanan, R. P. (Mayo de 2016): Stress profile of public elementary school teachers in Tacloban City Division: Inputs for a proposed classroom intervention program. En M. Plaisent (Presidencia), Multi-Disciplinary Scientific Conference. Conferencia llevada a cabo en el International Conference on Research in Social Sciences, Humanities and Education (SSHE-2016), Cebu, Philippines.

Pepe, A. y Addimando, L. (2013): "Comparison of occupational stress in response to challenging behaviours between general and special education Primary teachers in Northern Italy". International Journal of Special Education, 28 (1): 14-26.

Popov, S. et al. (2015): "The role of stressors at work and irrational beliefs in the prediction of teacher' stress". Primenjena Psihologija, 8 (1): 5-23.

Ramírez, T. et al. (2012): "Factores generadores de estrés y trabajo docente en Venezuela. Valoraciones diferenciales y repercusiones educativas”. Docencia Universitaria, 8 (1): 55-78.

Rangel, A. (2017): "Orientaciones pedagógicas para la inclusión de niños con autismo en el aula regular. Un apoyo para el docente". TELOS, Revista de Estudios Interdisciplinarios en Ciencias Sociales, 19 (1): 51-102.

Ruiz-Calzado, I. (2016): “Burnout en docentes de educación especial de Córdoba (España)”. Revista de Ciencias Humanas y Sociales, (12): 569-588.

Ruiz-Calzado, I. y Llorent, V. J. (2018): “El burnout en los profesionales de la educación que trabajan con personas con discapacidad en Córdoba (España). Influencia de las variables laborales”. Educación XX1, 21 (2): $373-393$.

Runes, D. D. (2010): “Enfermedades psicolaborales docentes”, en Sahili, L.F.A. (ed.): Psicología para el Docente. Consideraciones sobre los riesgos y desafíos de la práctica magisterial. México: Universidad de Guanajuato.

Samanamud, O. E. (2018): "Propiedades psicométricas de la Escala de Estrés Docente (ED-6) en Instituciones educativas estatales de secundaria del distrito de Trujillo". Searching-Humanities, 1 (1), 32.

Silva, L. S. y Valderrama, L. P. (2016): Creencias y prácticas de los agentes escolares sobre la inclusión educativa de los niños con discapacidad en la ciudad de Cartagena (Trabajo de Grado, Universidad de La Sabana, Colombia).

Sultana, B. et al. (2012): "The nature and impact of teacher stress in the private schools of Gilgit-Baltistan, Pakistan". International Journal of Academic Research in Progressive Education and Development, 1 (2): 64-84.

Venkatesh, B. y Ram, N. (2015): “Eustress: a unique dimension to stress management". Voice of Research, 4 (2): 26-29.

Vesga, L. S. y Vesga, J. M. (2015): “Una exclusión que se perpetúa: tensiones entre docentes, niños sordos y niños oyentes en escenarios escolares de Popayán”. Revista Virtual Universidad Católica del Norte, (46): 115-128. 
Vidal, J. B.et al. (2013): "Depressão como causa de afastamento do trabalho: Um estudo com professores do ensino fundamental”. Psico, 44 (2): 257-262.

Vieira, R. y Sacani, M. (Diciembre 2014): Estresse e inclusão escolar: influências e reflexos na práctica docente. VI Seminário Nacional de Educação Especial. Seminario llevado a cabo en el V Encontro de Pesquiadores em Educação Especial e Inclusão Escolar en la Universidad Federal de Uberlandia, Santa Mônica (Brasil). 